\title{
Location based Spatial Position Query Processing Environment for Position Queries using Energy Aware Clustering in VANET
}

\author{
Amit Neema \\ Radharaman Institute of Technology and Science \\ Computer Science and Engineering Department, \\ RGPV University Bhopa
}

\author{
Ashish Khare \\ Radharaman Institute of Technology and Science \\ Computer Science and Engineering Department, \\ RGPV University Bhopal
}

\begin{abstract}
Location-based spatial queries (LBSQs) refer to spatial queries whose answers rely on the location of the inquirer. Efficient processing of LBSQs is of critical importance with the ever-increasing deployment and use of mobile technologies. In a mobile environment, especially when the location of the objects change frequently and many queries are being generated, a centralized system cannot handle the query well. Hence in this work we propose a hybrid mechanism of handling the query by peer node called clusters. Nodes first listen to a broadcasting server which gets the information by beacon packets sent by the wireless nodes. In this paper, we focus on the propagation of local information originating in vehicles that is useful for other vehicles in the system. This type of activity has been described as Information Warning Functions (IWF).
\end{abstract}

We illustrate the appeal of our technique through extensive simulation results carried out using omnet++ Results shows that the system performance is independent of the topology.

\section{Keywords}

VANET, Clustering, Hybrid mobility, Flooding, Traffic regulation.

\section{INTRODUCTION}

\subsection{VANET}

A vehicular ad-hoc network or VANET is a technology that uses moving cars as nodes in a network to create a mobile network. VANET turns every participating car into a wireless router or node, allowing cars approximately 100 to 300 meters of each other to connect and, in turn, create a network with a wide range. As cars fall out of the signal range and drop out of the network, other cars can join in, connecting vehicles to one another so that a mobile internet is created. It is estimated that the first systems that will integrate this technology are police and fire vehicles to communicate with each other for safety purposes. Enabling data propagation in a VANET is challenging due to the existence of partitions between nodes described as network fragmentation. Existing routing protocols are based on the formation of an end-to-end path between source and destination or rely on a naming scheme and few exploit the mobility of nodes in a vanet. Resource discovery and naming are problematic under the presence of frequent and rapid arrivals and departures. For this paper, we concentrate on a pure ad hoc architecture absent any fixed infrastructure such as access points or satellite communication for data propagation. A common requirement for these vehicular networks is the existence of in-vehicle computing and communication capabilities and the assumption that geolocation is achieved via GPS. VANETs present a unique challenge in enabling message propagation. They are not nearly as constrained as MANETs in terms of available energy for computation and communication; rather, VANETs are characterized by extremely high mobility and rapidly changing topology. However, this mobility is constrained in motion due to the existence of roadways and can therefore be cleverly exploited for message propagation. There are some existing routing protocols that have been explored for application in this domain but they have not used these characteristics; mobility, direction of motion, and location information, to enhance the performance of the routing protocols. There are several challenges to adopting existing routing protocols from the field of MANETs [6, 7]. First, the network can frequently form partitions preventing end-to-end communication strategies. Second, resource discovery and naming are problematic as the vehicles are in general unreliable and frequent arrivals and departures occur.

\section{PRESENT SYSTEM}

The existing system is centralized. A server accumulates all the information and does not employ broadcasting. Rather, the peer nodes makes explicit query which is responded by the server. As wireless data by nature is broadcasted to open air, such a centralized system adds extra overhead in the wireless environment and processing. Beside every independent queries are to be processed by the server. In situations like VANET where such a system is extensively used, scaling the application for wider reachibility is a problem.

1. Existing techniques cannot be used effectively in a wireless broadcast environment, where only sequential data access is supported.

2. It may not scale to very large user populations. In an existing system to communicate with the server, a client must most likely use a fee-based cellular-type network to achieve a reasonable operating range.

3. Users must reveal their current location and send it to the server, which may be undesirable for privacy reasons. 


\section{PROPOSED SYSTEM}

\section{Overall Block Diagram}

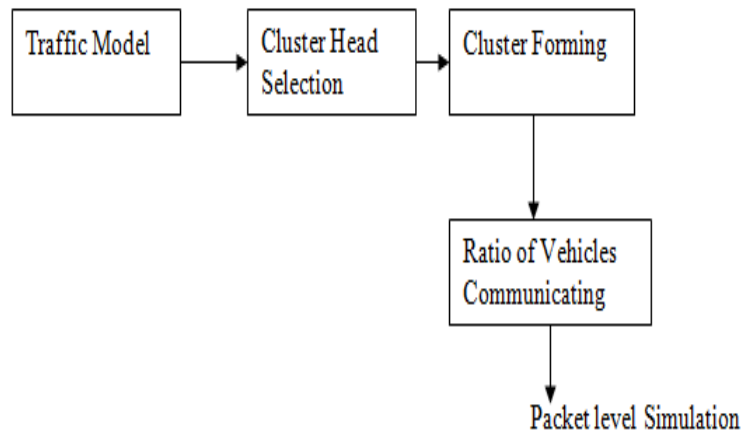

Fig 1: Cluster Head Selection

\subsection{Connectivity Aware Topology Control}

An ad hoc network called as connected if and only if there is at least a path between each pair of mobile nodes. Connectivity thus depends on the existence of routes. it is affected by changes in the topology due to mobility: link failure, route updates, rerouting, etc. in order to achieve connectivity, we need to determine the locations of the mobile routers that maximize the number of covered mobile nodes. The deployment must ensure a connected backbone.

\subsection{The Parameters}

We consider:

$>\mathrm{N}$ mobile stations (MS) to be covered by $\mathrm{M}$ mobile routers (MR), all located on a flat rectangular field of surface $\mathrm{AxB}$.

> each mobile node MSi is represented by the geometrical point $\mathrm{Pi}$ with coordinates (xi, yi),

D each mobile router $\mathrm{MRj}$ is represented by the geometrical point Qj with coordinates (aj, bj).

$>\mathrm{Rr}$ denotes the mobile router transmission range,

$>\mathrm{Rm}$ denotes the mobile station transmission range,

$>\mathrm{d}(\mathrm{J}, \mathrm{K})$ denotes the Euclidian distance between geometrical points $\mathrm{J}$ and $\mathrm{K}$.

In order to be covered by a router, the distance between a mobile station and its closest router must be less than $\mathrm{Rm}$. Two mobile routers are neighbors (i.e. adjacent in the backbone network) if the distance between them is less than Rr.

We define:

$\mathrm{Xmin}=\min (\mathrm{xi}) \quad, \quad \mathrm{xmax}=\max (\mathrm{xi})$

$$
1<=\mathrm{i}<=\mathrm{N} \quad 1<=\mathrm{i}<=\mathrm{N}
$$

\section{I. $\quad \mathrm{YMIN}=\mathrm{MIN}(\mathrm{YI}) \quad ; \quad \mathrm{YMAX}=\operatorname{MAX}(\mathrm{YI})$}

$$
1<=\mathrm{i}<=\mathrm{N} \quad 1<=\mathrm{i}<=\mathrm{N}
$$

Variables:

For $\mathrm{i}=1, \ldots, \mathrm{N}$ and $\mathrm{j}=1, \ldots, \mathrm{M}$, (aj, bj) denote the router's coordinates,

Let $\lambda \mathrm{i}, \mathrm{j}=\left\{\begin{array}{c}1 \text { if } \mathrm{d}(\mathrm{pi}, \mathrm{Qj})<=\mathrm{Rm} \\ 0 \quad \text { otherwise }\end{array}\right.$

In other words, $\lambda \mathrm{i}, \mathrm{j} \square=1$ iff the mobile station MSiis covered by the mobile router MRj (ensures the connectivity between mobile node MSi and mobile router MRj)

For $\mathrm{i}, \mathrm{j}=1, \ldots, \mathrm{M}$,

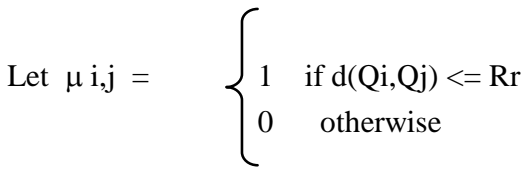

$\mu \mathrm{i}, \mathrm{j}=$ iff MRi is an adjacent router of MRj in the mobile routers' backbone (ensures the connectivity between two mobile routers $\mathrm{MRi}$ and $\mathrm{MRj}$ )

The backbone network may be represented as a graph whose vertices represent the mobile routers, and whose the adjacency matrix is $(\mu \mathrm{i}, \mathrm{j}) ; 1<=\mathrm{i}, \mathrm{j}<=\mathrm{M}$

For $\mathrm{I}=1, \ldots \mathrm{N}$, Let $\tau \mathrm{i}=1$ iff $\mathrm{MSi}$ is covered by at least one mobile router, that is, if there exists at least one router $\mathrm{MRj}$ for which $\lambda i, j=1$ To check the backbone (formed by mobile routers) connectivity, we will test whether it is possible to create a route from any mobile router MRs $(s=2, \ldots, M)$ to the router number 1 (MR1) Hence, we define zsi,j as zsi,j =1 if the route from the router $\mathrm{s}$ to the router number 1 goes through the link $(i, j)$, otherwise $z s i, j=0$

\section{RESULTS}

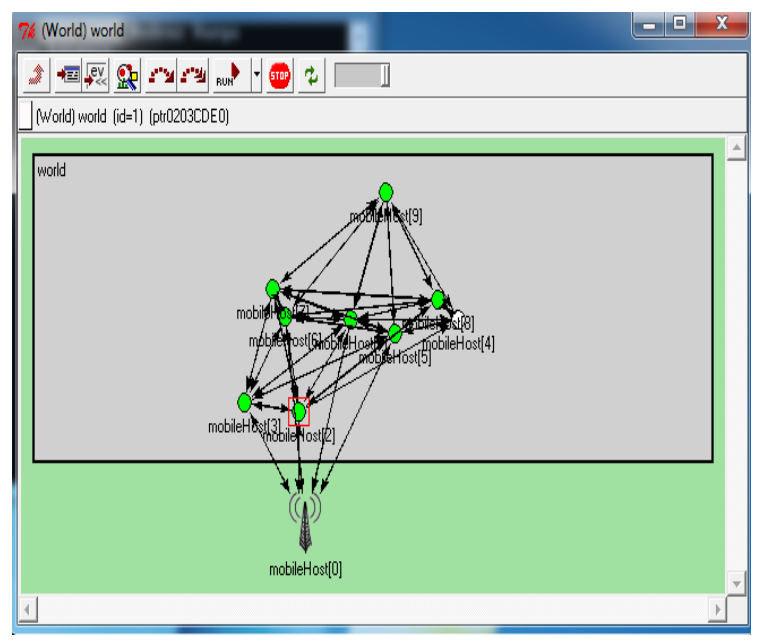

Fig 2: Simulation Environment 
No of Nodes v/s PDR, Throughput, Latency, Control Overhead

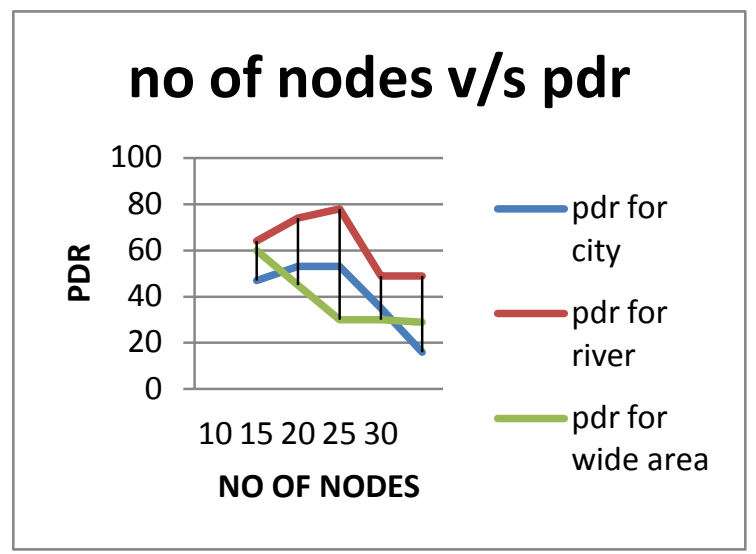

Fig 3

The Graph shows that Number of Node increases, pdr comes down in city area due to congestion. But in country side the performance is always good. The performance of the system is satisfactory for 25 nodes. Hence the spatial query can be considered to be optimum.

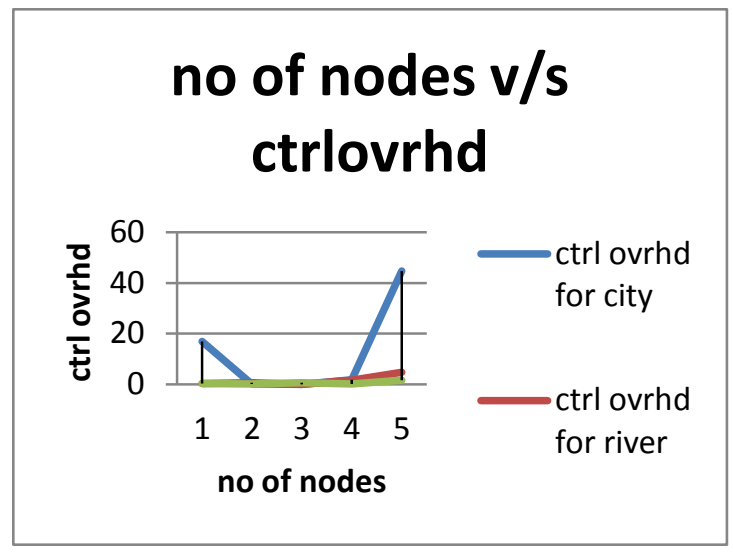

Fig 4

Control overhead increases with increase in city traffic. However for other two topologies, increase in control overhead is restricted. This proves that for the system to be widely deployable in cities, we need to introduce better congestion avoidance mechanism.

\section{no of nodes $v / s$ tp}

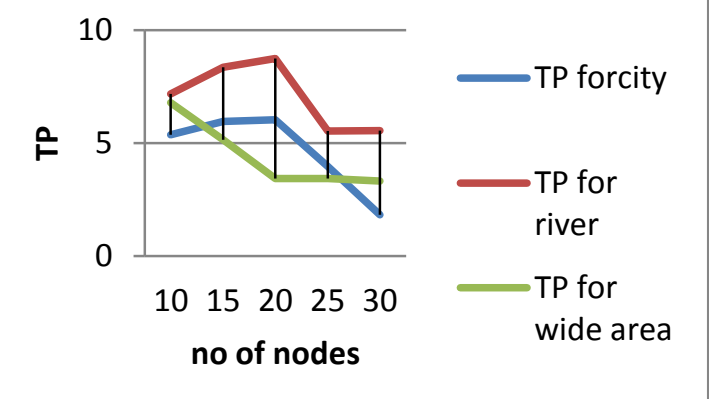

Fig 5

Throughput is about $40 \%$ of maximum channel capacity which is considered to be very good. Now for city traffic the throughput is low under heavy traffic which can be improved by introducing number of cluster head nodes.

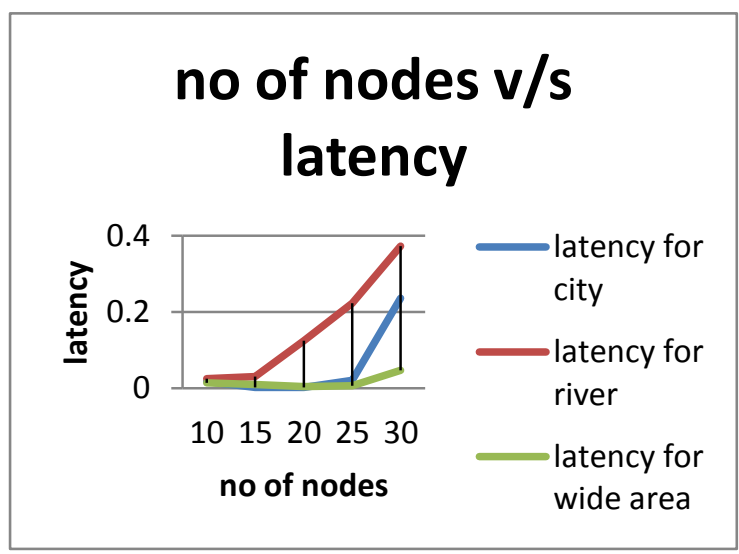

Fig 6

The graph shows that the average latency is independent for low traffic but is highly affected under congestion.

\section{CONCLUSION}

VANET offers several benefits to organizations of any size. While such a network does pose certain safety concerns (for example, one cannot safely type an email while driving), this does not limit vanet's potential as a productivity tool. In VANET integrates on multiple ad-hoc networking technologies such as WIFI IEEE 802.11Pand ZIGBEE for easy, accurate, effective and simple communication between vehicles on dynamic mobility. The system was tested with different architecture like riverside, city block, wide area and demonstrated that the performance of the system is independent of the type of locations and also that the system is quite scalable and the scalability does not affect the performance of the system. The system can be further improved by incorporating region wise clustering scheme where several cluster heads can cooperate for optimum result. 


\section{REFERENCES}

[1] N. Beckmann, H.-P. Kriegel, R. Schneider, and B. Seeger. The $\mathrm{R}^{*}$-Tree: An Efficient and Robust Access Method for Points and Rectangles. In SIGMOD Conference, pages 322-331, 1990.

[2] C.-Y. Chow, H. V. Leong, and A. T. S. Chan. Distributed Group-based Cooperative. Caching in a Mobile Broadcast Environment. In Mobile Data Management, pages 97-106, 2005.

[3] M. de Berg, M. van Kreveld, M. Overmars, and O. Schwarzkopf. Computational Geometry Algorithms and Applications (2nd Edition). Springer, 2000.

[4] A. Guttman. R-Trees: A Dynamic Index Structure for Spatial Searching. In SIGMOD Conference, pages 4757, Boston, Massachusetts, June 18-21, 1984.

[5] G. R. Hjaltason and H. Samet. Distance Browsing in Spatial Databases. ACM Trans. Database Syst., 24(2):265-318, 1999.
[6] T. Imielinski, S. Viswanathan, and B. R. Badrinath. Data on Air: Organization and Access. IEEE Trans. Knowl. Data Eng., 9(3):353-372, 1997.

[7] N. Roussopoulos, S. Kelley, and F. Vincent. Nearest Neighbor Queries. In SIGMOD Conference, pages 7179, 1995.

[8] T. K. Sellis, N. Roussopoulos, and C. Faloutsos. The R+Tree: A Dynamic Index for Multi-Dimensional Objects. In VLDB, pages 507-518, 1987.

[9] L. Yin and G. Cao. Supporting cooperative caching in ad hoc networks. IEEE Trans. Mob. Comput., 5(1):77-89, 2006.

[10] J. Zhang, M. Zhu, D. Papadias, Y. Tao, and D. L. Lee. Location-based Spatial Queries. In SIGMOD Conference, pages 443-454, 2003.

[11] B. Zheng, W.-C. Lee, and D. L. Lee. Spatial Queries in Wireless Broadcast Systems. Wireless Networks, 10(6):723-736, 2004 